\title{
Bevacizumab-associated glomerular microangiopathy
}

\author{
Fermin Person $\mathbb{1}^{1} \cdot$ Markus M. Rinschen ${ }^{2,3} \cdot$ Silke R. Brix $^{4} \cdot$ Sonia Wulf ${ }^{1} \cdot$ Maria de las Mercedes Noriega ${ }^{1}$. \\ Wilfried Fehrle ${ }^{1}$ - Jessica Schmitz ${ }^{5} \cdot$ Anke Schwarz $^{6} \cdot$ Philipp Ivanyi $^{7}$ - Oliver M. Steinmetz ${ }^{4} \cdot$ Linda Reinhard $^{4}$. \\ Elion Hoxha ${ }^{4}$ Peter F. Zipfel $\mathbb{1}^{8} \cdot$ Jan Hinrich Bräsen ${ }^{5} \cdot$ Thorsten Wiech $^{1}$
}

Received: 11 June 2018 / Revised: 5 November 2018 / Accepted: 6 November 2018 / Published online: 14 December 2018

(c) United States \& Canadian Academy of Pathology 2018

\begin{abstract}
Bevacizumab is a humanized monoclonal IgG1 antibody, which neutralizes vascular endothelial growth factor and is used for treating multiple cancer types. As a known and frequent adverse event, this therapy can lead to renal damage including proteinuria and nephrotic syndrome. In a retrospective approach, we analyzed 17 renal biopsies from patients receiving bevacizumab treatment. We observed a distinctive histopathological pseudothrombotic pattern different from the previously reported thrombotic microangiopathy. Since this pattern includes some features similar to acute and chronic thrombotic microangiopathy, focal segmental glomerulosclerosis and cryoglobulinemic membranoproliferative glomerulonephritis, biopsies with these diagnoses were included for comparison. Clinical, laboratory, light microscopic, immunohistochemical (including a proximity ligation assay), proteomic and electron microscopic features were assessed. Nephrotic syndrome was present in 15 of the 17 bevacizumab-treated patients. All 17 displayed a patchy pattern of variably PAS-positive hyaline pseudothrombi occluding markedly dilated glomerular capillaries in their biopsies. Mass spectrometry-based proteome analysis revealed a special protein pattern demonstrating some features of thrombotic microangiopathy and some of cryoglobulinemic glomerulonephritis, including a strong accumulation of $\mathrm{IgG}$ in the pseudothrombi. Proximity ligation assay did not show interaction of IgG with $\mathrm{Clq}$, arguing for accumulation without classic pathway complement activation. In contrast to thrombi in thrombotic microangiopathy cases, the hyaline pseudothrombi did not contain clusters of CD61positive platelets. Electron microscopy of bevacizumab cases did not show fibrin polymers or extensive loss of podocyte foot processes. Even though cases of bevacizumab-associated microangiopathy share some features with thrombotic microangiopathy, its overall histopathological pattern is quite different from acute or chronic thrombotic microangiopathy cases. We conclude that bevacizumab therapy can lead to a unique hyaline occlusive glomerular microangiopathy, likely arising from endothelial leakage followed by subendothelial accumulation of serum proteins. It can be diagnosed by light microscopy and is an important differential diagnosis in cancer patients with nephrotic syndrome.
\end{abstract}

These authors contributed equally: Jan Hinrich Bräsen, Thorsten Wiech

Electronic supplementary material The online version of this article (https://doi.org/10.1038/s41379-018-0186-4) contains supplementary material, which is available to authorized users.

Thorsten Wiech

t.wiech@uke.de

1 Institute of Pathology and Nephropathology Section, University Hospital Hamburg Eppendorf, Hamburg, Germany

2 Department II of Internal Medicine and Center for Molecular Medicine Cologne (CMMC), University of Cologne, Cologne, Germany

3 Cologne Excellence Cluster on Cellular Stress Responses in Aging-Associated Diseases (CECAD), University of Cologne, Cologne, Germany

\section{Introduction}

Bevacizumab is a humanized anti-vascular endothelial growth factor monoclonal mouse antibody [1] inhibiting neoangiogenesis. It is used in the treatment of a variety of neoplastic

4 III. Department of Medicine, University Medical Center HamburgEppendorf, Hamburg, Germany

5 Nephropathology Unit, Institute of Pathology, Hannover Medical School, Hannover, Germany

6 Clinic for Nephrology, Hannover Medical School, Hannover, Germany

7 Department of Hematology, Hemostasis, Oncology and Stem Cell Transplantation, Hannover Medical School, Hannover, Germany

8 Leibniz Institute for Natural Product Research and Infection Biology, Jena, Germany 


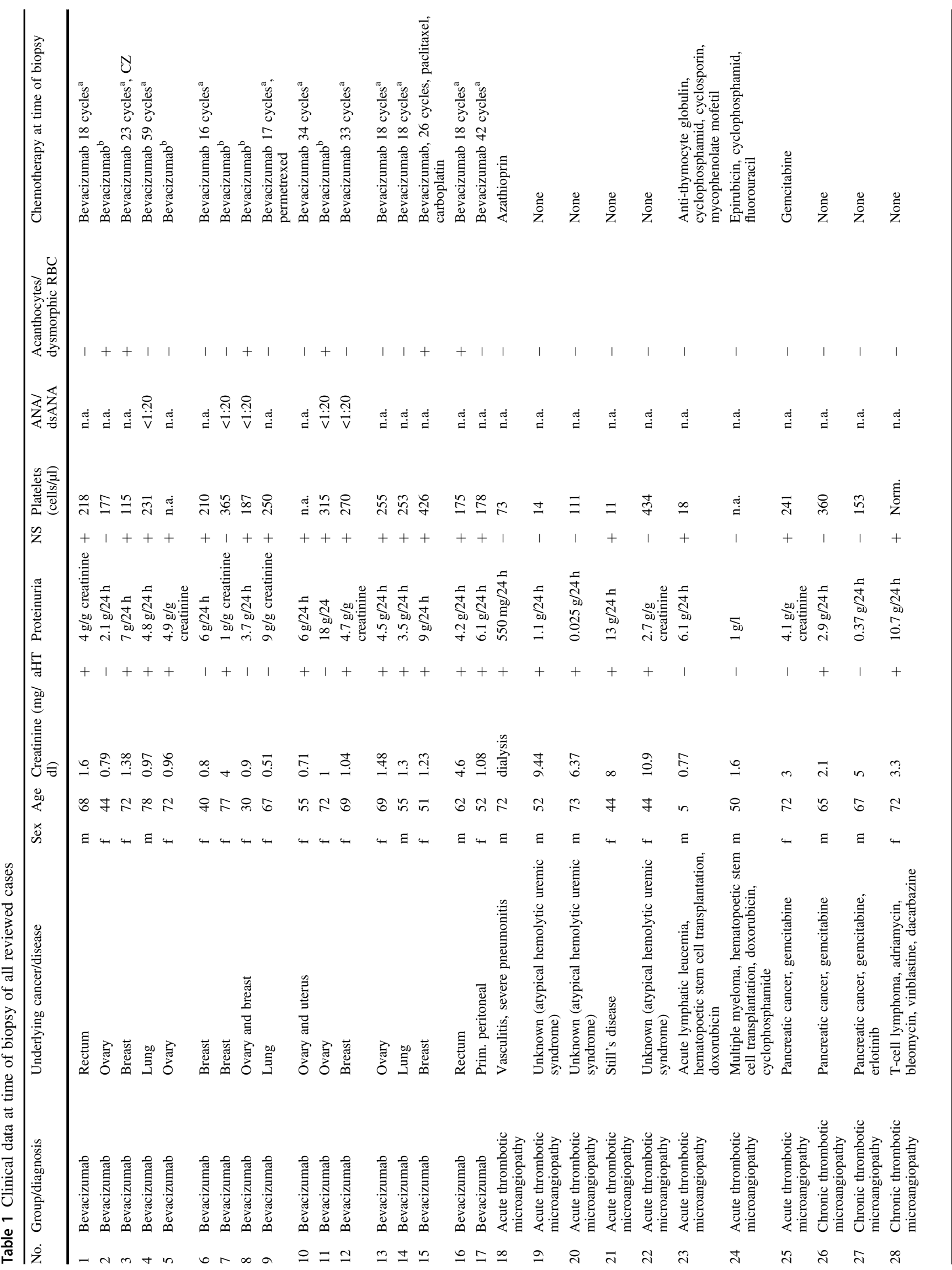




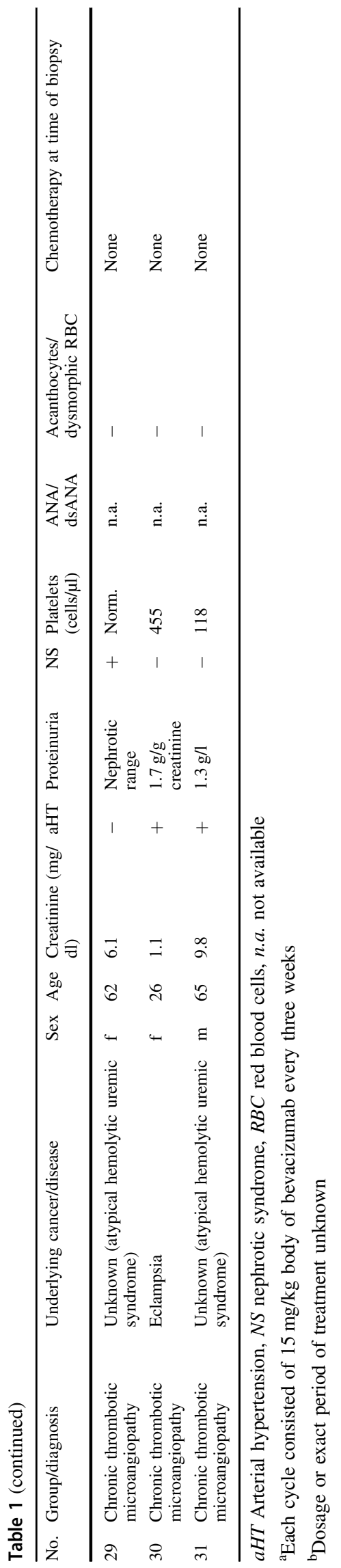

and non-neoplastic diseases such as breast cancer, colon carcinoma, glioblastoma, renal cell carcinoma, lung cancer [2], and age-dependent macular degeneration [3]. Proteinuria up to the nephrotic range has been described as an adverse event in various studies. Urinary excretion of more than 300 $\mathrm{mg}$ of protein per day was measured in 15 to $26.5 \%$ of bevacizumab-treated patients. The incidence of nephrotic range proteinuria $(\geq 3.5 \mathrm{~g} / 24 \mathrm{~h}$ ) has been reported as $0.7-7 \%$ in this setting [4-6]. The morphologic correlate of bevacizumab-associated renal adverse event has been investigated in previous studies and has included: acute interstitial nephritis [7, 8], glomerular lesions (including minimal change disease [9]) and, most notably, thrombotic microangiopathy [10-15]. It is unknown how often proteinuria is caused by bevacizumab-induced thrombotic microangiopathy, but it is believed that acute thrombotic microangiopathy occurs in only a minority of the cases with nephrotic range proteinuria during bevacizumab therapy [10-15].

Several previous studies have tried to clarify the possible pathophysiological mechanism leading to bevacizumabassociated renal damage and proteinuria. It has been suggested that the lack of soluble vascular endothelial growth factor [4] leads to the downregulation of nephrin in podocytes and thereby loss of endothelial fenestration $[6,16]$. Furthermore, it has been shown that vascular endothelial growth factor inhibition decreases local complement regulators in the kidney such as complement factor $\mathrm{H}$ [17], which is a further possible damage mechanism in glomeruli. In studies involving mouse or rat models, glomerulosclerosis and loss of podocyte foot processes were observed [18, 19]. A mouse model of thrombotic microangiopathy was established by blocking renal vascular endothelial growth factor expression [13]. Similar effects were seen in transgenic mice presenting the human FcyRIIa (hFcR) receptor on their platelets [20]. In monkey eyes, immune complexes were observed in the presence of heparin after bevacizumab administration [21]. However similar immune complexes have, up to now, never been observed in human specimens. Furthermore, the exact mechanism leading to proteinuria during bevacizumab therapy could not be sufficiently explained so far.

To our knowledge, this is the first report of a distinctive injury pattern in kidneys of bevacizumab-treated proteinuric patients, sharing some features of thrombotic microangiopathy. The aim of this study is to describe the characteristics of bevacizumab-associated microangiopathy in detail.

\section{Materials and methods}

\section{Patients and biopsies}

In the years 2007-2017, 12 cases with proteinuria during bevacizumab treatment were identified in the archives of 
the Nephropathology Section of the Institute of Pathology, University Hospital Hamburg-Eppendorf. Five additional cases were identified in the archives of the Institute of Pathology at Hannover Medical School. Details on clinical presentation and medications of the selected patients at the time of renal biopsy are summarized in Table 1 (more detail including cryoglobulinemic membranoproliferative glomerulonephritis is provided in Supplemental Table 1). All patients received bevacizumab treatment because of cancer. Fifteen of the 17 patients $(88 \%)$ had nephrotic range proteinuria of $>3 \mathrm{~g} / \mathrm{d}$. None of the patients had clinical evidence for systemic lupus erythematosus. For comparison we included eight cases of acute thrombotic microangiopathy (cases 18-25) caused by atypical hemolytic uremic syndrome or chemotherapy, as well as six cases with chronic thrombotic microangiopathy (26-31). Further, additional comparison for purposes of differential diagnosis was performed with biopsies revealing cryoglobulinemic membranoproliferative glomerulonephritis and secondary focal segmental glomerulosclerosis (Supplemental Tables 1-3). For validation of the mass spectrometry performed, we included five cases of fibrillary glomerulonephritis (IgGpositive control) and five zero hour transplant biopsies (negative control) in the analysis. We also included one case of immune complex membranoproliferative glomerulonephritis as a positive control for the $\mathrm{IgG} / \mathrm{C} 1 \mathrm{q}$ proximity ligation analysis.

The study was approved by the local ethics committees in both Hamburg and Hannover (Ethics commission Hamburg, PV 5541; Ethics commission Hannover Medical School, No. 3606-2017). All work was carried out in accordance with the Declaration of Helsinki.

\section{Light microscopy}

For light microscopic evaluation, PAS stained sections were examined and the pattern of injury was determined. The following criteria were analyzed: mesangial and endocapillary hypercellularity (both defined according to the Oxford classification of IgA nephropathy 2016 [22]), size and structure of intracapillary thrombi or pseudothrombi, number of normal versus segmentally or globally sclerosed glomeruli, glomerular collapse, and double contours of the peripheral glomerular capillary walls. Since massive dilatation and occlusion of the glomerular capillaries was observed in the bevacizumab cases, the maximal diameter of the glomerular capillary containing a thrombus/pseudothrombus was measured for every affected glomerulus in all three patient groups. Slides were digitized using an Axio Scan Z1 scanner (Zeiss, Jena, Germany) and diameter measurements of the slides were performed using the ZEN viewer software (Zeiss, Jena, Germany).

\section{Immunohistochemistry}

In addition to standard diagnostic immunohistochemical markers (IgA, IgG, IgM, C1q, C3, and fibrinogen/fibrin), we performed staining for IgG subclasses $1-4$, CD61 for platelets, and CD34 for endothelial cells. Sections $(1 \mu \mathrm{m})$ of the archival paraffin blocks were cut and deparaffinized in xylene followed by a descending series of ethanol. For $\mathrm{IgA}, \mathrm{IgG}, \operatorname{IgM}, \mathrm{C} 3, \operatorname{IgG1}-4, \mathrm{C} 1 \mathrm{q}$, fibrinogen/fibrin, and CD61, enzyme-induced epitope retrieval was performed for $15 \mathrm{~min}$ with proteinase (protease P-8038, SigmaAldrich, St. Louis, Missouri, USA) at $40{ }^{\circ} \mathrm{C}$. Subsequently, primary antibodies specific for $\operatorname{IgA}(1: 8000)$, IgM (1:3000), fibrinogen/fibrin $(1: 12,000)$, C3 (1:3000), C1q (1:1500), CD61 (1:50) (all DAKO, Eching, Germany), and IgG (1:7500; Dianova, Hamburg, Germany) were applied for $30 \mathrm{~min}$ at $40^{\circ} \mathrm{C}$, and bound antibodies were then visualized manually using a standard APAAP protocol. Primary antibodies for IgG1 (1:100), IgG2 (1:40,000), IgG3 (1:100), IgG4 (1:1000) (all SouthernBiotec, Alabama, USA) were incubated at $4{ }^{\circ} \mathrm{C}$ for $16 \mathrm{~h}$. For CD34 the slides were exposed to heat-induced epitope retrieval for $15 \mathrm{~min}$ in an autoclave at $120^{\circ} \mathrm{C}$ in $\mathrm{pH} 6.2$ citrate buffer. Afterwards, the slides were incubated at $4{ }^{\circ} \mathrm{C}$ for $16 \mathrm{~h}$ with the primary antibody (clone QBEnd/10, Novocastra, Newcastle, UK, 1:4000). IgG1-4, CD34 and CD61 antibodies were visualized manually using the Zytomed detection Kit POLAP-100 rabbit/mouse (Zytomed Systems, Berlin, Germany).

\section{Proximity ligation assay}

Five cases of bevacizumab-associated glomerulopathy and one case of immune complex membranoproliferative glomerulonephritis were chosen for analysis. As described above, sections $(1 \mu \mathrm{m})$ of the archival paraffin blocks were cut and deparaffinized in xylene followed by a descending series of ethanol. For pretreatment, protease (protease P8038, Sigma-Aldrich, St. Louis, Missouri, USA) was applied for $30 \mathrm{~min}$ at $40^{\circ} \mathrm{C}$. Afterwards, IgG (1:7500; mouse, Dianova, Hamburg, Germany) and subsequently C1q (1:1500; rabbit, DAKO, Eching, Germany) were applied for $30 \mathrm{~min}$ each at $40^{\circ} \mathrm{C}$. Then secondary antibodies against mouse and rabbit antibodies (Duolink ${ }^{\circledR}$ In Situ proximity ligation assay Probes, Sigma-Aldrich, Darmstadt, Germany) were added. According to protocol, the brightfield detection system (Duolink ${ }^{\circledast}$ In Situ Detection Reagents Brightfield, Sigma-Aldrich, Darmstadt, Germany) was applied to the slides [23]. The density of the glomerular signals was analyzed using image analysis software (CellDetector, HSAnalysis, Karlsruhe, Germany) (Supplemental Fig. 1). 


\section{Electron microscopy}

The portion of the renal biopsy selected for electron microscopy during standard diagnostic work-up was transferred from $4 \%$ formaldehyde into a cacodylate buffer together with sucrose for $10 \mathrm{~min}$ at $80^{\circ} \mathrm{C}$. Afterwards, osmiumtetroxyde was applied for $2 \mathrm{~h}$. Next, the specimen was washed in cacodylate buffer plus sucrose two times for $5 \mathrm{~min}$. Subsequently, the sample was contrasted with uranyl acetate for $1 \mathrm{~h}$. The specimen was then put into ethanol baths with rising ethanol concentrations for $5 \mathrm{~min}$ each, followed by tert-Methyl tert-butyl ether (MTBE) twice for 5 min each, MTBE plus epoxide mixture (in a 1:3 dilution). Afterwards, the specimens were embedded in an epoxide mixture at $60{ }^{\circ} \mathrm{C}$ for $48 \mathrm{~h}$ and then at $100^{\circ} \mathrm{C}$ for $11 \frac{1 / 2}{\mathrm{~h}}$. Semithin and ultrathin sections were cut on a Reichelt-Jung Ultracut-E701704 microtome. Grids were purchased from Polyscience (Cham, Germany). The grids were then analyzed using electron microscopes (EM 109 and EM 902, Zeiss, Jena, Germany) equipped with digital electron microscope cameras (Tröndle, Moorenweis, Germany). One glomerulus from each case was analyzed.

\section{Serological analyses}

Since the observed hyaline pseudothrombi in the bevacizumab group revealed immunohistochemical positivity for immunoglobulins, one hypothesis was that immune complexes could contain newly formed antibodies against bevacizumab. The presence or absence of such antibodies was evaluated by ELISA in bevacizumab patient no. 1 . Bevacizumab $(5 \mu \mathrm{g} / \mathrm{ml})$ was immobilized to the surface of a microtiter plate. Following washing, patient serum (bevacizumab patient no. 1), normal human serum (1:500 dilution) from a healthy individual (NHS\#1), and two Deaphemolytic-uremic syndrome patients with autoantibodies to Factor H (HS\#2 and HS\#3) were added [24]. After washing, bound human $\mathrm{IgG} 1, \operatorname{IgG} 2$, IgG3, or IgG4 were detected with specific mouse antibodies (Dako, each at 1:5500 dilution) followed by peroxidase coupled anti-mouse serum [25].

\section{Sample preparation for mass spectrometry-based proteomics analysis}

Due to the prevalence of thrombi or pseudothrombi in the bevacizumab-associated cases and in thrombotic microangiopathy cases, we decided to analyze the composition of the glomerular thrombi on a proteomic level using highly sensitive mass spectrometry. To the best of our knowledge, no glomerular thrombi or pseudothrombi were analyzed on the proteomic level before. The glomeruli of five cases each of bevacizumab-associated glomerulopathy, acute thrombotic microangiopathy, zero hour biopsies and, as a positive control, fibrillary glomerulonephritis were harvested from 7 $\mu \mathrm{m}$ thick slides and mounted on 1.0 PEN MembraneSlides ${ }^{\circledR}$ (Zeiss, Jena, Germany) using a PALM MicroBeam laser microscope (ZEISS, Jena, Germany). The glomeruli of each case were collected into a separate tube. Between 40 and 112 sections of glomeruli per case could be harvested. Afterwards, the individual samples were processed using the ultrasensitive protocol for sample preparation and mass spectrometry exactly as previously described [26]. In brief, glomeruli were lysed in $40 \mu 18 \%$ SDS buffer, boiled and incubated with $25 \mathrm{U}$ Benzonase and $1 \mu \mathrm{l} 200 \mathrm{mM}$ DTT for $30 \mathrm{~min}$ at $37^{\circ} \mathrm{C}$. The lysates were alkylated using $2 \mu \mathrm{l} 200$ mM IAA in the dark, then a SP3 digestion was performed [27]. All samples were analyzed on a Q-Exactive Plus mass spectrometer (Thermo Fisher Scientific, Bremen, Germany) coupled to an EASY-nLC 1000 (Thermo Fisher Scientific) using $1 \mathrm{~h}$ gradients as previously described [26].

\section{Bioinformatic analysis}

Acquired mass spectrometry raw data were blasted using MaxQuant v 1.5.4.3 against the human reference proteome database, including common contaminants (downloaded from www.uniprot.org in January 2017), using the target decoy approach [28]. The bevacizumab protein sequence (obtained from www.drugbank.ca (DB00112) [29]) was added to the database. Default stringent parameters were used, including a protein, peptide and PSM false discovery rate of $<0.01$. Quantification was performed by label-free quantification intensities (LFQ) using the MaxQuant LFQ algorithm [30]. Match between run was enabled. The output file ("proteingroups.txt") was filtered and contaminants, reverse hits and proteins identified by site only were removed. Protein intensities were $\log _{2}$ transformed. Expression was normalized against zero hour biopsies and expressed as a $\log _{2}$ ratio. Statistical significance was determined by a two-tailed $t$-test followed by correction for multiple testing using the significance analysis of microarrays approach first introduced by Tusher et al. (FDR < 0.01 , s0 $=0.1$ ) [31]. The twenty most strongly increased and significant proteins were reported. Overlap of potential bevacizumab derived peptides with various endogenous immunoglobulin peptides were manually validated via the blastp software and sequence alignments.

\section{Results}

\section{Clinical and laboratory findings}

Of the 17 patients undergoing bevacizumab therapy, 15 displayed a nephrotic syndrome (88\%, Table 2). Three of the eight acute thrombotic microangiopathy patients (38\%) 


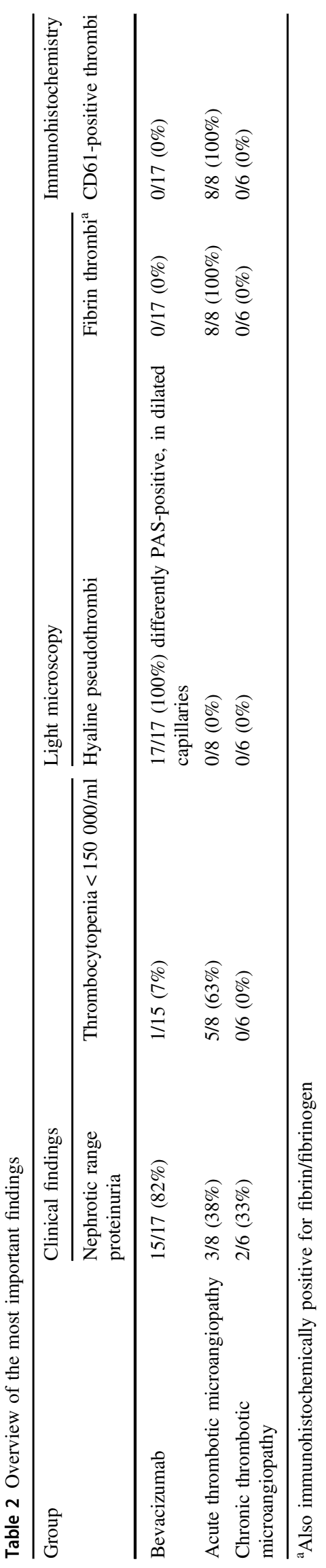

suffered from nephrotic range proteinuria (defined as proteinuria $>3 \mathrm{~g} / 24 \mathrm{~h}$ or $>3 \mathrm{~g} / \mathrm{g}$ creatinine). The serum creatinine levels in the bevacizumab group were normal or slightly elevated (mean $1.4 \mathrm{mg} / \mathrm{dl}, \mathrm{Sd} \pm 0.8 \mathrm{mg} / \mathrm{dl}$ ), whereas in the acute thrombotic microangiopathy cases creatinine was usually highly elevated (mean $5.7 \mathrm{mg} / \mathrm{dl}, \mathrm{Sd} \pm 3.9 \mathrm{mg}$ / dl) or the patient required hemodialysis (1/8). Apart from one patient in the bevacizumab group who had mild thrombocytopenia $(115.000 / \mu \mathrm{l})$, all remaining patients in this group had normal platelet counts. This is in contrast to the acute thrombotic microangiopathy cases in which five out of the eight cases had severe thrombocytopenia (Table 2, for more detail Table 1). Furthermore chronic thrombotic microangiopathy patients also showed a clinical constellation that was very distinct from bevacizumab cases, with a highly elevated creatinine (mean $4.6 \mathrm{mg} / \mathrm{dl}, \mathrm{Sd} \pm 3.1$ $\mathrm{mg} / \mathrm{dl}$ ). Five of the seven cryoglobulinemic membranoproliferative glomerulonephritis patients $(5 / 7,71 \%)$ showed nephrotic range proteinuria (more detail see Supplemental Table 1).

\section{Light microscopic findings}

All 17 biopsies from proteinuric patients under bevacizumab therapy showed a unique patchy pattern of variably PAS-positive pseudothrombi. These pseudothrombi often occluded markedly dilated capillary lumens with direct contact to the capillary walls. Double contours of the glomerular basement membrane occurred in other areas (Fig. 1, Supplemental Figure 3). The preglomerular arteries were spared in all of the bevacizumab cases. None of the bevacizumab cases showed signs of glomerular collapse such as wrinkled GBM or collapsed glomerular tufts (typical examples see Supplemental Figure 3). In only 2 of the 17 bevacizumab cases segmental glomerular sclerosis could be detected (Table 3). No glomerulus in any of the bevacizumab cases fulfilled the criteria for collapsing focal segmental glomerulosclerosis according to the Columbia classification [32]. All eight acute thrombotic microangiopathy cases showed classical signs of thrombotic microangiopathy in their biopsies, including mesangiolysis, endothelial swelling, fibrin thrombi containing platelets, fragmented red blood cells, and in some cases ischemic collapse of glomerular tufts. In none of these acute or chronic thrombotic microangiopathy cases could PASpositive hyaline pseudothrombi be found (Fig. 1). Neither the bevacizumab cases nor the acute or chronic thrombotic microangiopathy cases showed a diffuse mesangial or endocapillary hypercellularity.

The maximum diameter of glomerular capillaries containing a thrombus/pseudothrombus was $18.4 \mu \mathrm{m} \quad \mathrm{Sd} \pm$ $6.38 \mu \mathrm{m})$ in the cases of bevacizumab-associated glomerulopathy (case 1-17) and $15.5 \mu \mathrm{m}(\mathrm{Sd} \pm 5.5 \mu \mathrm{m})$ in the acute 


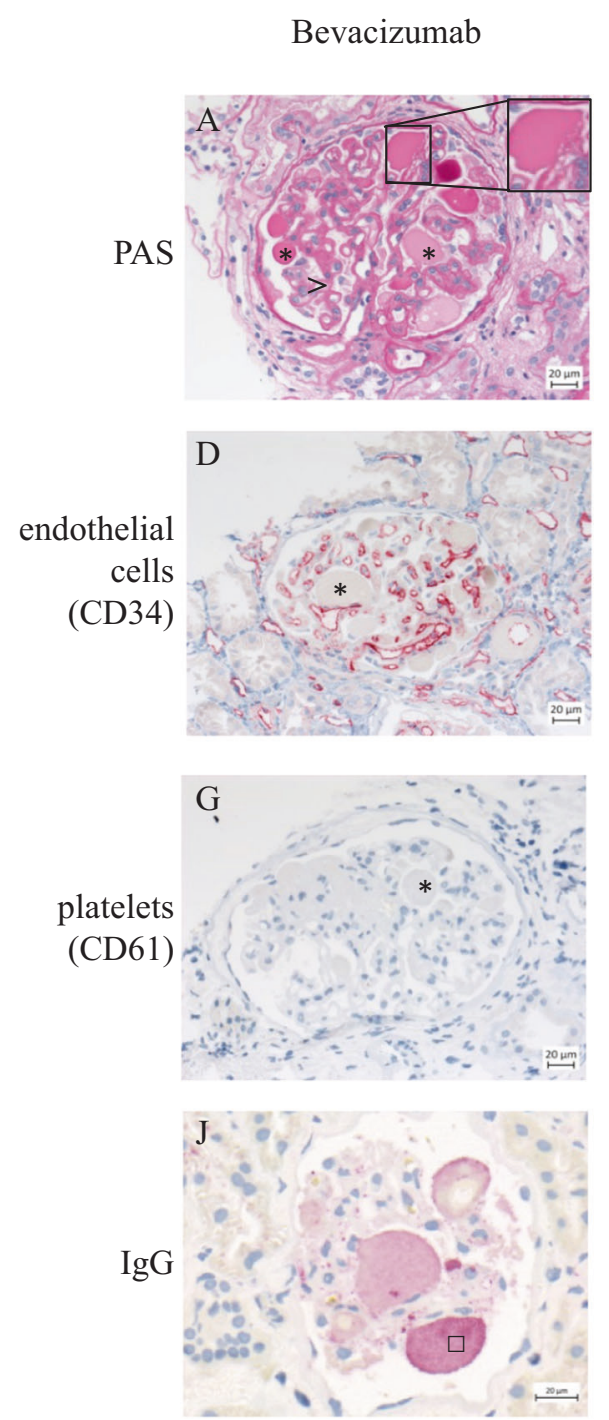

Fig. 1 Representative stains of bevacizumab-associated glomerular, acute and chronic thrombotic microangiopathy cases. a, d, g, f Representative PAS, CD34, CD61, and IgG-stains of bevacizumabassociated glomerular microangiopathy cases, displaying differently strongly PAS-positive hyaline pseudothrombi in dilated capillaries (asterisk) with double contours (greater than symbol) and loss of CD34 positive endothelial cells (asterisk). Note absence of platelets (asterisk)

thrombotic microangiopathy cases (case 18-25, Table 2). Affected glomerular capillaries of bevacizumab cases were significantly more dilated than the capillaries of acute thrombotic microangiopathy cases $(p<0.05)$. An overview of the light microscopic findings is given in Table 2 (more details in Supplemental Table 2).

The affected capillaries in cryoglobulinemic membranoproliferative glomerulonephritis (case 37-43) also proved to be much smaller than in bevacizumab cases, measuring $12.7 \mu \mathrm{m}(\mathrm{Sd} \pm 3.34 \mu \mathrm{m})(p<0.001)$. A comparison of bevacizumab, acute thrombotic microangiopathy and cryoglobulinemic membranoproliferative glomerulonephritis cases can be found in Supplemental Figure 4.
Acute thrombotic microangiopathy
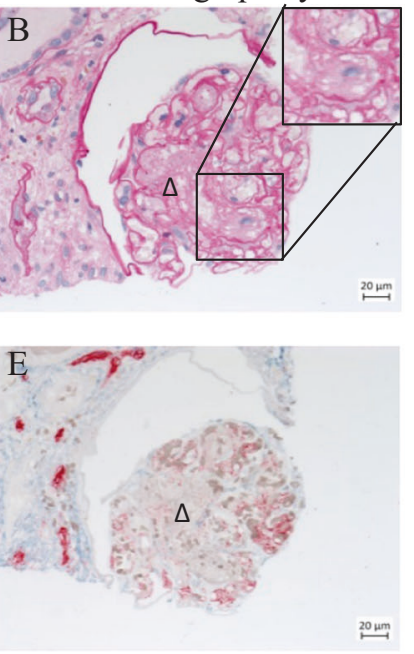

$\stackrel{20 \mathrm{~m}}{\mathrm{M}}$
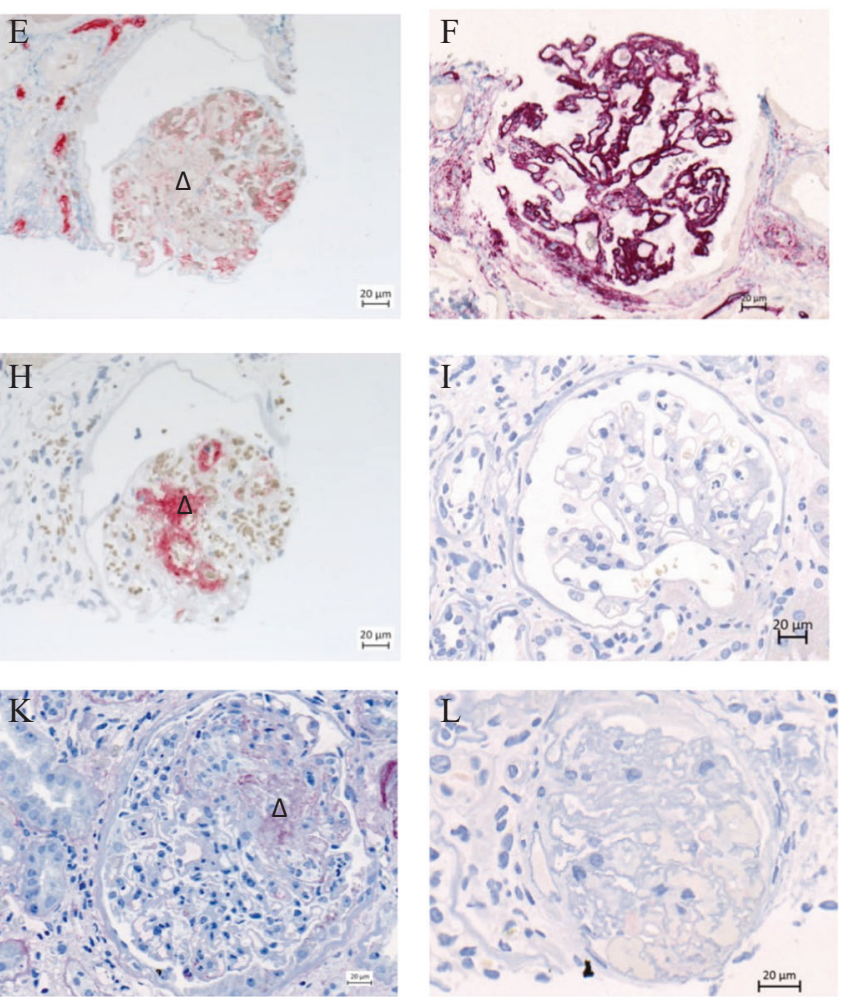

and positivity of pseudothrombi for $\operatorname{IgG}$ (empty box). b, e, h, k Representative PAS, CD34, CD61, and IgG-stains of acute thrombotic microangiopathy cases. See thrombi containing platelets in dilated glomerular capillaries (empty triangle). Marked loss of CD34 positive endothelial cells around the thrombi. Nearly no positivity for IgG in thrombi (empty triangle). c, f, i, I Representative PAS, CD34, CD61, and IgG-stains of chronic thrombotic microangiopathy (I)

\section{Immunohistochemical findings}

CD34 staining displayed at least a focal loss of glomerular endothelial cells in areas of thrombi or pseudothrombi in all groups. In the bevacizumab cases, no thrombi containing CD61-positive platelets were detected. In contrast, all thrombotic microangiopathy cases displayed accumulation of CD61-positive platelets in "real" thrombi of the glomerular capillaries and in some preglomerular arterioles. In all bevacizumab cases, only a weak nonspecific positivity for fibrinogen/fibrin was observed in the described pseudothrombi. In most cases, these were also variably positive for IgA, IgM, and IgG (Supplemental Table 2). Staining for 


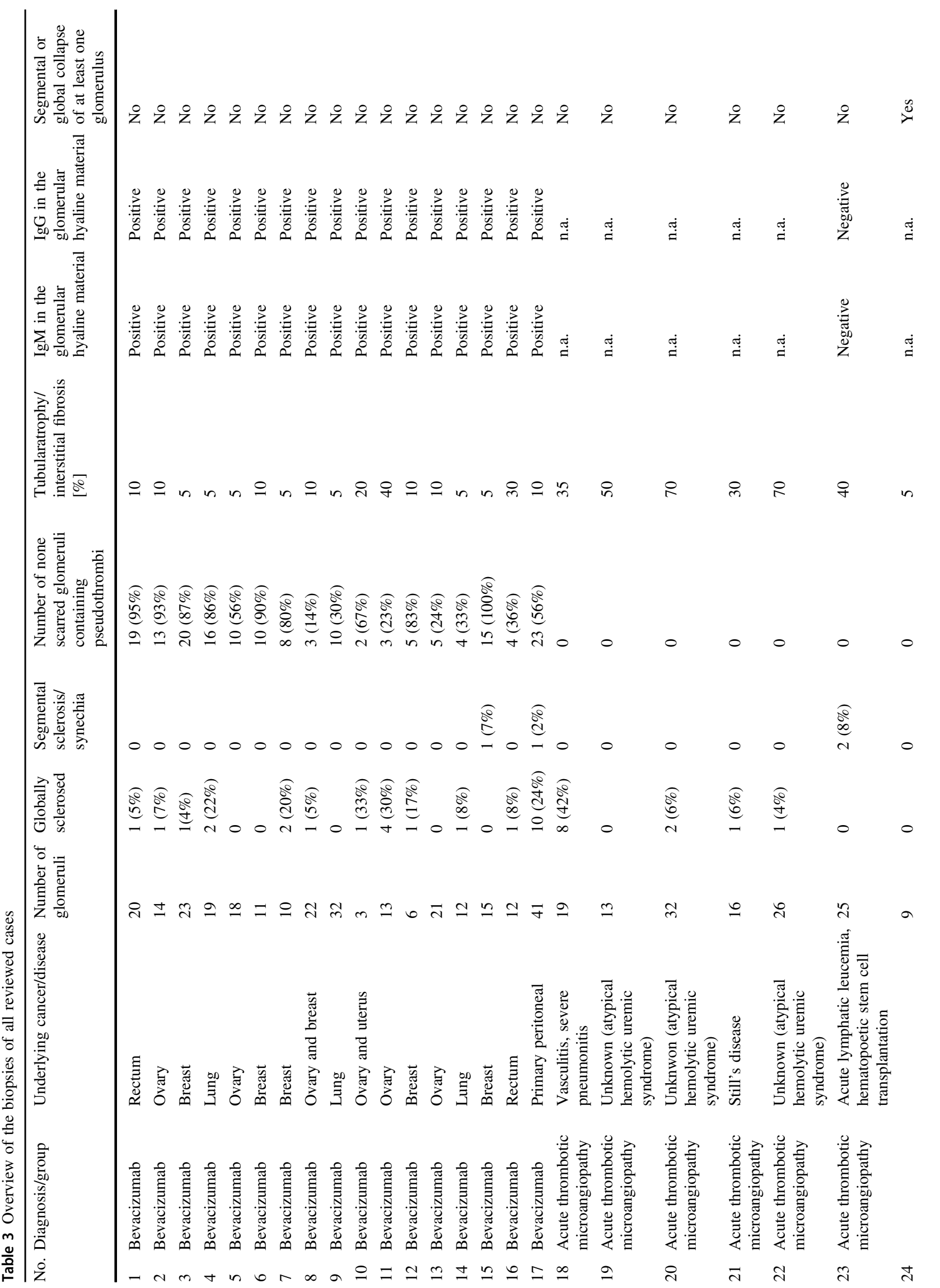




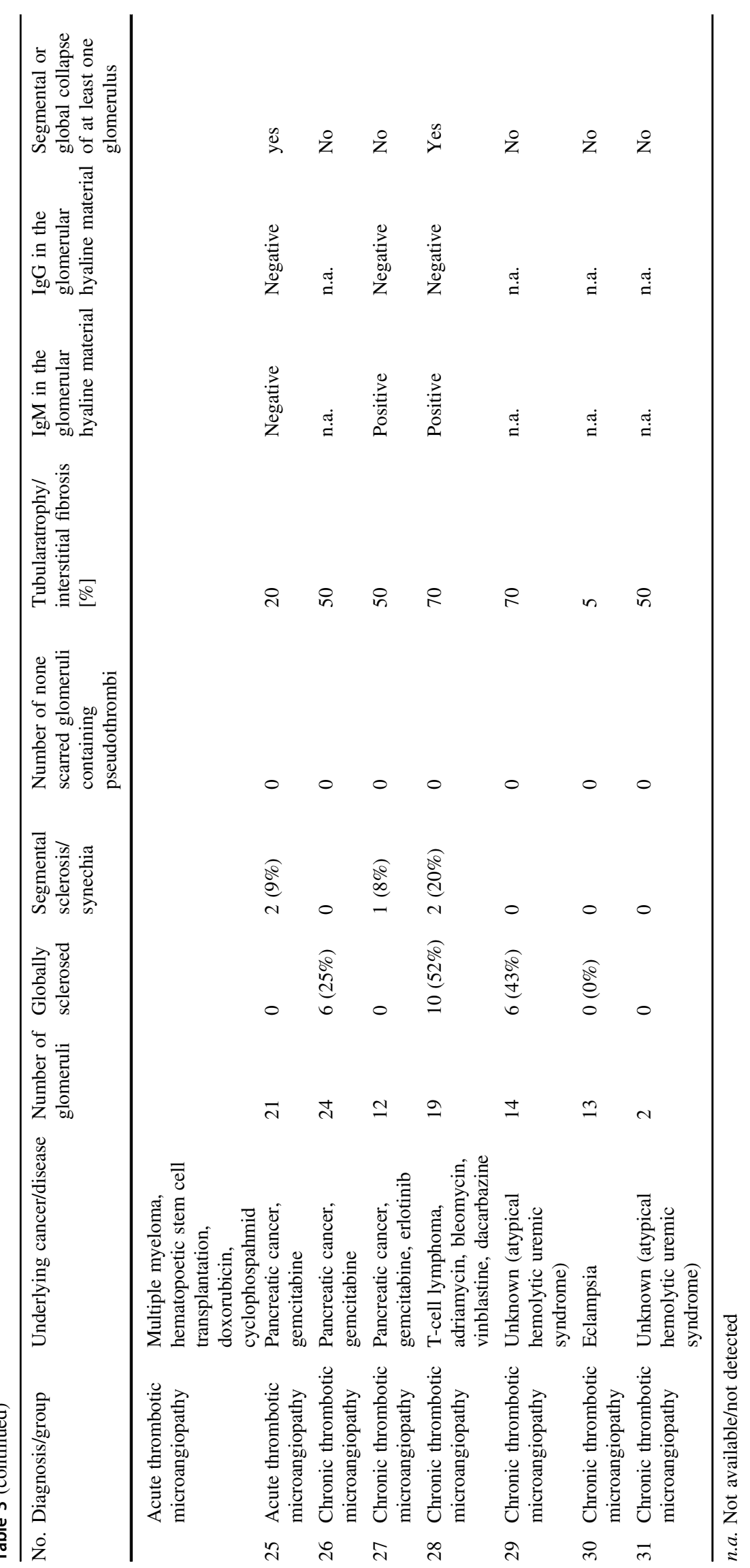




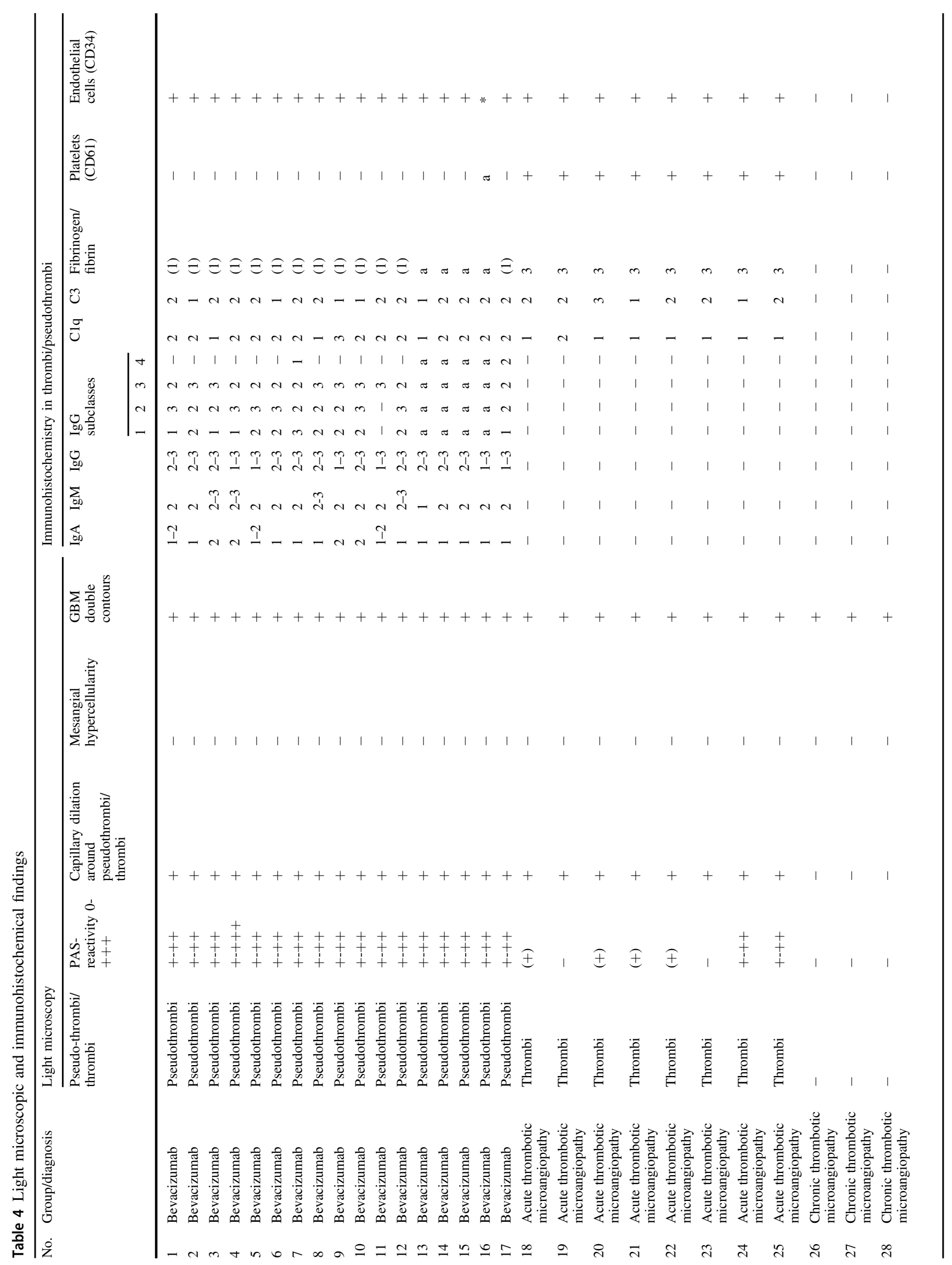


the IgG subclasses revealed positivity for IgG1-3 in all but one of the cases, which was only positive for IgG3 (case 11, Table 3). Case 11 did not show monoclonal kappa or lambda stain. Positivity for $\mathrm{IgG} 4$ was detected in one case (case 7, Table 4). The typical histologic and immunohistochemical findings are displayed in Fig. 1.

\section{Proximity ligation assay findings}

The positive control (immune complex membranoproliferative glomerulonephritis) showed abundant dot-like proximity ligation signals, indicating a distance between $\operatorname{IgG}$ and $\mathrm{C} 1 \mathrm{q}$ of less than $40 \mathrm{~nm}$, thus allowing interaction between the two molecules [33]. In contrast, the cases of bevacizumabassociated glomerulopathy showed almost no signals (Supplemental Fig. 1). This suggests that no classical complement pathway activation takes place in bevacizumab-associated glomerulopathy, making an immune complex-mediated genesis of the glomerulopathy in this setting highly unlikely.

\section{Electron microscopic findings}

Electron-dense pseudothrombi could be found in the majority of the cases with bevacizumab nephropathy (12/16, $75 \%)$. These were absent in all thrombotic microangiopathy cases $(0 / 13,0 \%)$. No platelets or fibrin polymers were identified in any pseudothrombi using ultrastructural analyses. In bevacizumab cases, all pseudothrombi showed highly electron-dense material which lacked diagnostic secondary structures at high magnification. Endothelial cells were nearly completely absent in regions of highly distending pseudothrombi. Additional mesangial deposition of electron-dense material could be found in all bevacizumab cases. In contrast, the acute thrombotic microangiopathy cases lacked electron-dense mesangial deposits. Subendothelial deposits could be found in both diseases: ten of the 17 bevacizumab-associated glomerular microangiopathy cases $(10 / 17,59 \%)$ and 5 of 13 thrombotic microangiopathy cases $(5 / 13,38 \%)$ showed subendothelial deposits. Subepithelial deposits were present in 8 of 17 bevacizumab cases $(8 / 17,47 \%)$, whereas they were absent in all eight acute thrombotic microangiopathy cases. Extensive (more than $75 \%$ ) loss of the podocytic foot processes was not observed in bevacizumab cases, whereas it was present in three of thirteen $(3 / 13,23 \%)$ thrombotic microangiopathy cases. Table 6 and Supplemental Table 4 summarize the electron microscopic findings shown in Fig. 2.

\section{Serological results}

In the analyzed serum of bevacizumab patient no. 1 , no antibodies against bevacizumab could be detected in the ELISA test (see Supplemental Fig. 2). 
Fig. 2 Representative electron microscopic pictures of glomeruli affected by bevacizumab-associated glomerular microangiopathy. a Variably electron-dense pseudothrombi (right arrow) and glomerular double contours (greater than symbol). b Pseudothrombus in dilated capillary (asterisk). Note largely preserved podocyte foot processes (right arrow) and loss of endothelial cells (greater than symbol)
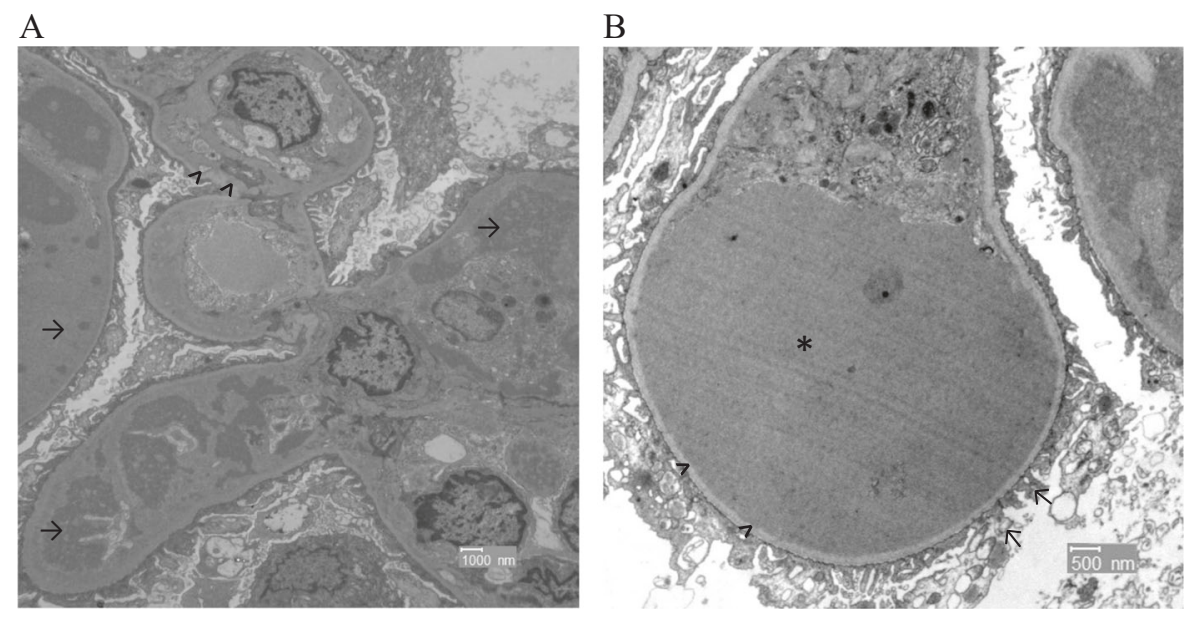

\section{Mass spectrometry results}

An overview of the mass spectrometric findings relative to the zero hour transplant biopsies, including the 20 most strongly increased proteins, can be found in Table 5. We expressed all protein levels relative to zero hour transplant biopsies of unaffected kidneys. As a positive control, we included fibrillary glomerulonephritis cases, in which DNAJB9 was the most abundant protein [34]. In acute thrombotic microangiopathy cases, fibrin (indicative of fibrin-thrombus formation) was the most strongly increased protein. In contrast, the most strongly increased protein in bevacizumab-associated cases was $\mathrm{C} 4 \mathrm{~b}$-binding protein alpha chain, followed by Alpha- 2 macroglobulin, fibrinogen, Ig alpha-1 chain and Ig heavy chain. Because of the great similarity between human $\operatorname{IgG}$ molecules and the bevacizumab molecule, being a humanized antibody, bevacizumab itself could not be detected with certainty.

\section{Discussion}

Bevacizumab is known to cause damage to endothelial cells that can lead to a pattern resembling acute thrombotic microangiopathy [13]. Here, we describe another unique light microscopic pattern in bevacizumab-treated patients suffering from nephrotic syndrome. This pattern of bevacizumab-associated microangiopathy, manifested exclusively in glomeruli, is associated with nephrotic range proteinuria and exhibits some features of acute and chronic thrombotic microangiopathy (e.g., loss of endothelial cells and double contours of the basement membranes) as well as of cryoglobulinemic membranoproliferative glomerulonephritis (e.g., deposition of immunoglobulines) Table 6.

In contrast to cryoglobulinemic membranoproliferative glomerulonephritis, bevacizumab-associated glomerular microangiopathy exhibits a marked dilatation of the capillaries with only mild endocapillary and mesangial hypercellularity. These cases also show a unique proteome composition, which is distinct from that of cryoglobulinemic glomerulonephritis.

Bevacizumab-associated glomerular microangiopathy can also be distinguished morphologically from acute thrombotic microangiopathy by several aspects. It lacks fibrin precipitates in the light microscopy and electron microscopy as well as clotted CD61-positive platelets immunohistochemically, and instead shows variably intense IgG positivity in the pseudothrombi. In addition, bevacizumab cases show a unique proteome composition that is distinct from acute thrombotic microangiopathy cases. The potential differential diagnostic consideration of chronic glomerular thrombotic microangiopathy can be distinguished by the fact that bevacizumab cases show hyaline pseudothrombi, which are different from the secondary segmental sclerosis sometimes seen in thrombotic microangiopathy cases [35]. As none of the bevacizumab cases shows signs of glomerular collapse (according to the definition given by the Columbia classification [32]), the pattern seems to be quite distinct from the glomerular collapse that is regularly seen in chronic thrombotic microangiopathy cases [35]. In keeping with these morphological differences, it is important to note that bevacizumab-only glomerulopathy cases also present a clinical picture distinct from that of acute thrombotic microangiopathy cases.

As shown immunohistochemically, bevacizumabassociated pseudothrombi exhibit a rather high abundance of $\operatorname{IgG}$ (in contrast to thrombotic microangiopathy) which, however, does not seem to be directly associated with complement activation, as shown by our proximity ligation assay results. Considering the latter, it is unlikely that immune complex-mediated complement activation (as seen in cryoglobulinemic membranoproliferative glomerulonephritis) plays a causative role in bevacizumab-associated microangiopathy, at least regarding the classical pathway. 


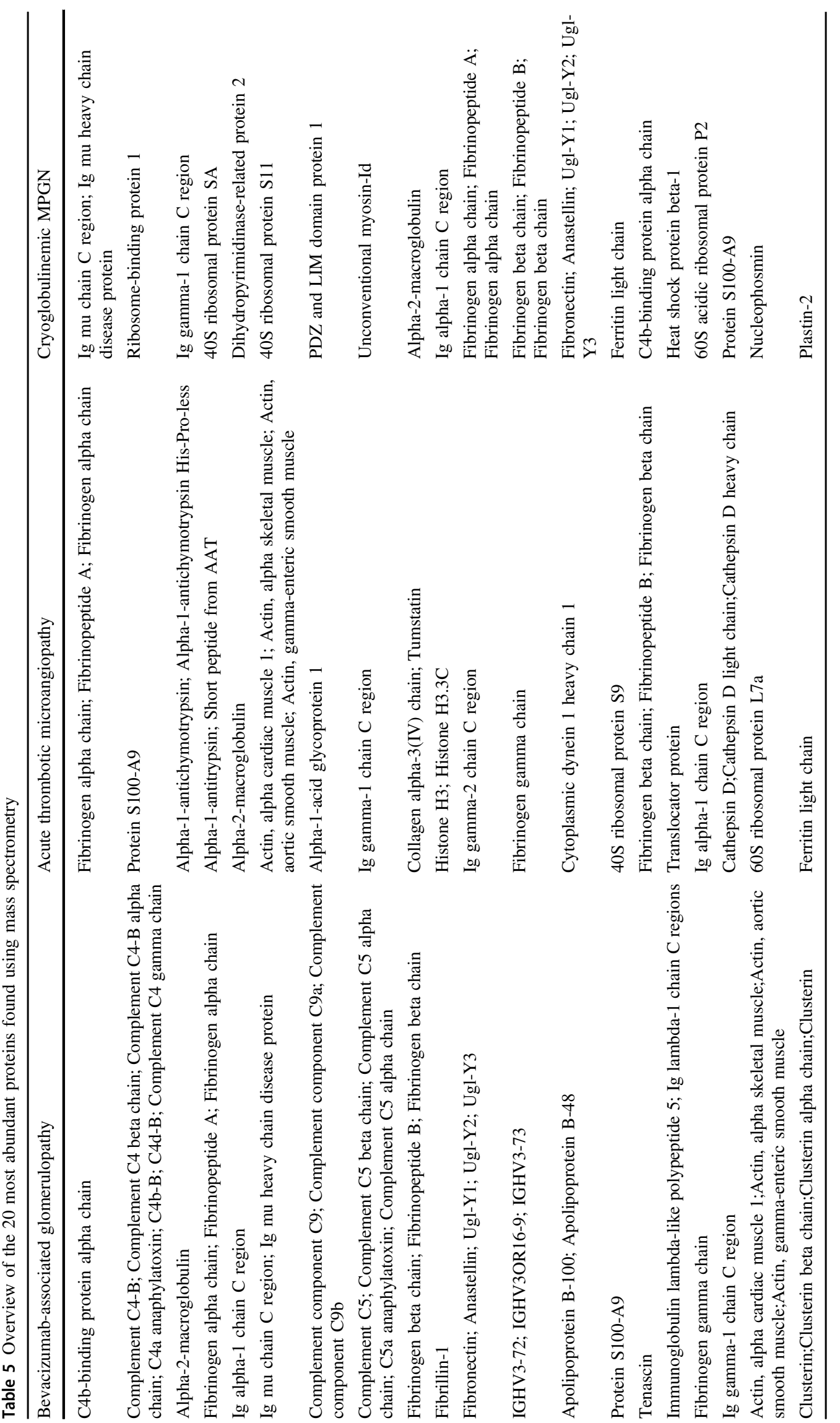




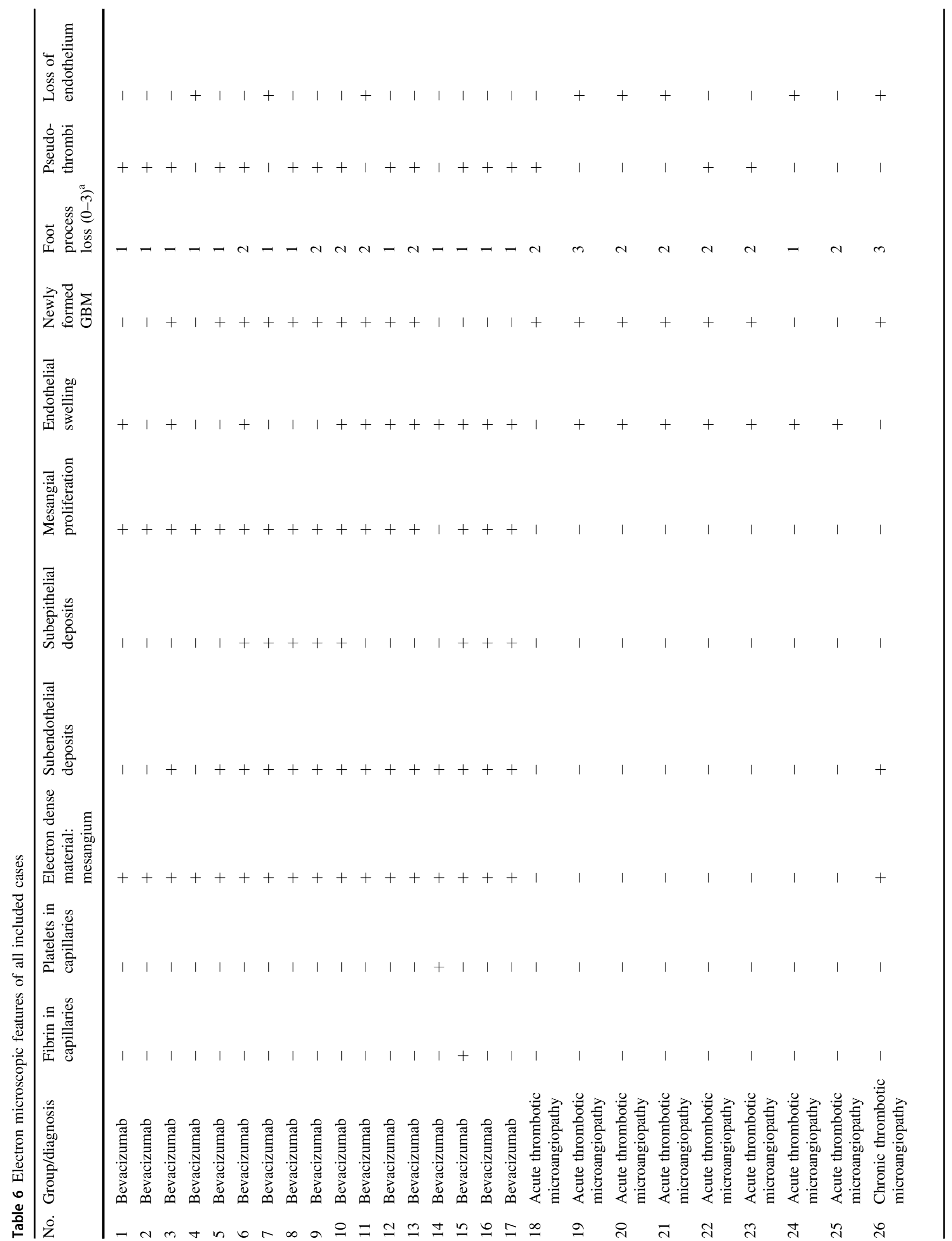




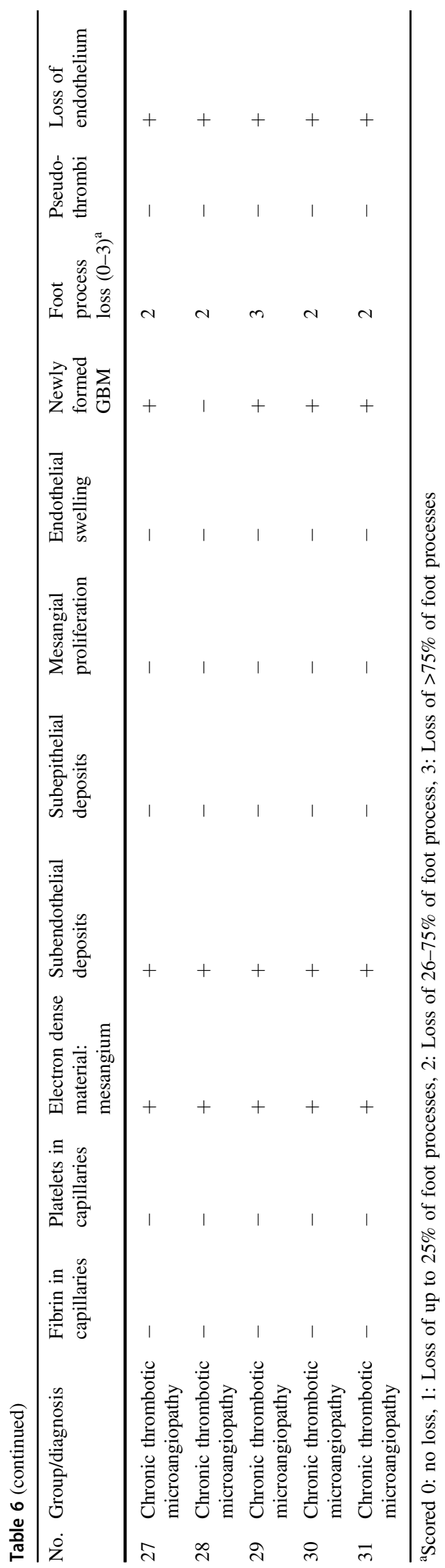

Despite this clear presence of IgG in bevacizumabassociated glomerulopathy lesions, albeit at variable concentrations, it remains unclear which role these IgG molecules play. We could prove neither significant entrapment of the bevacizumab molecule itself nor antibody production directed against it.

Exhibiting a unique histologic and proteomic pattern, it is likely that the pathogenesis of bevacizumab-associated glomerular microangiopathy differs from that of acute thrombotic microangiopathy. The common final path of different thrombotic microangiopathy forms is endothelial cell death and the subsequent precipitation of fibrin and platelets as well as the occurrence of fragmented red blood cells [36]. In contrast, bevacizumab microangiopathy lacks fibrin/platelet thrombi as well as fragmented red blood cells, evaluated both by light microscopy and proteomic analysis. Instead, large hyaline pseudothrombi completely occlude markedly dilated capillary lumens, sometimes giving the appearance of microaneurysmata.

The exact pathophysiologic mechanisms leading to the formation of these pseudothrombi still need to be elucidated. Since the patients analyzed here had no evidence for systemic lupus erythematosus or cryoglobulinemia two pathways are conceivable:

The first hypothesis is that immune complexes form pseudothrombi within the glomerular capillaries and lead to mechanical destruction of endothelial cells. Our ELISA analysis did not reveal antibodies against bevacizumab (only one patient was available for evaluation) and only very small amounts of vascular endothelial growth factor were found in the glomeruli by immunohistochemistry and mass spectrometry (data not shown). This argues against large amounts of immune complexes consisting of either bevacizumab with anti-bevacizumab antibodies or of bevacizumab with vascular endothelial growth factor. Furthermore, proximity ligation assay analysis did not show classical complement activation and no significant hypercellularity could be observed in the lesions.

These findings favor the second hypothesis, namely that vascular endothelial growth factor inhibition caused by bevacizumab might lead to an increased endothelial permeability for serum proteins with subsequent deposition and accumulation in the subendothelial space, without the formation of true immune complexes. The accumulated material might prevent activation of the coagulation system despite endothelial cell loss, leading to the absence of actual fibrin precipitates and/or platelet thrombi. These pseudothrombi are strongly positive not only for $\mathrm{IgG} 1$, which might at least in part represent bevacizumab itself, but also for $\mathrm{IgG}$, IgG3 and in one case also IgG4, as well as $\operatorname{IgM}$ and even IgA. This favors a nonspecific accumulation rather than formation of specific immune complexes. Our mass spectrometry data support that bevacizumab-associated 
glomerulopathy is pathophysiologically more related to immunoglobulin deposition whereas thrombotic microangiopathy is not. Additionally, altered regulation of the complement system by the podocytes due to vascular endothelial growth factor inhibition might further contribute to this effect [17].

Although oncological patients often receive different agents in parallel, our collectives included cases of gemcitabine or doxorubicin monotherapy-associated thrombotic microangiopathy and we selected renal biopsies from patients receiving single chemotherapy for comparison. Interestingly we observed during routine diagnostics other side effects of small molecule tyrosine kinase inhibitor inhibitors of vascular endothelial growth factor activation (e.g., Pazopanib). None of these small molecule inhibitors related cases demonstrated thrombotic microangiopathy or bevacizumab-associated glomerulopathy-like manifestations, but rather showed acute tubular damage and tubulointerstial inflammation (data not shown). This reflects a completely divergent damage pattern of different chemotherapeutic agents, even though they target the same pathway. The particular reaction pattern or manifestation most likely depends on conditions of the micromilieu in the patients' glomeruli. For instance, all affected patients of our cohort were treated with at least 16 cycles of bevacizumab over 12 months before becoming symptomatic, so the changes described could likely be interpreted as a cumulative effect. Furthermore, remission of nephrotic syndrome in those patients with accessible data ranged from 2 to 8 months, which might represent dissolution of the accumulated material (data not shown).

Given the context of accumulation of hyaline and hyaline material, we compared our bevacizumab-associated glomerulopathy cases to cryoglobulinemic membranoproliferative glomerulonephritis as well as cases with chronic thrombotic microangiopathy and focal segmental glomerulosclerosis, including the collapsing variant (see Supplemental Tables 1, 2 and 3), and still found a distinctive morphology which correlated clinically with a prominent proteinuric (and less function-deteriorating) phenotype.

Irrespective of the exact pathophysiologic mechanism, we describe a unique histomorphologic pattern of bevacizumabassociated glomerulopathy in a collection of 17 patients. This pattern consists of a characteristic alternating between strongly PAS-(and immunoglobulin-) positive and virtually PAS-negative hyaline pseudothrombi occluding markedly dilated glomerular capillaries. As earlier publications have shown that patients with high proteinuria and hypertension demonstrate a better anti-tumor response under bevacizumab treatment [37], it might be speculated that patients with high proteinuria have a higher endothelial susceptibility to antivascular endothelial growth factor medication.

In summary we describe, for the first time, a distinct bevacizumab-associated glomerulopathy with nephrotic range proteinuria different from the known bevacizumab associated form of thrombotic microangiopathy. Recognizing this pattern can be important for the differential diagnosis in cancer patients suffering from a nephrotic syndrome.

Acknowledgements We thank Ulrike Langbehn, Ina Löschmann and Mahtab Taleb-Naghsh for excellent technical assistance.

Funding This study has been funded by the Deutsche Forschungsgesellschaft (DFG), SFB 1192 project B6 to TW and PFZ and by the Dr. Werner Jackstädt-Stiftung to JHB and JS. MMR was supported by the DFG (RI 2811-1/1).

\section{Compliance with ethical standards}

Conflict of interest The authors declare that they have no conflict of interest.

Ethical approval The study was conducted in accordance with the Declaration of Helsinki and the German legislation for the use of archived tissue specimens and associated clinical information. The clinical data were retrieved and the histological samples were collected and analyzed with the endorsement of the local ethics committee of the local board of physicians at Hamburg and Hannover (Ethics commission Hamburg, PV 5541; Ethics commission Hannover Medical School, No. 3606-2017).

\section{References}

1. Ferrara N, Hillan KJ, Gerber H-P, Novotny W. Discovery and development of bevacizumab, an anti-VEGF antibody for treating cancer. Nat Rev Drug Discov. 2004;3:391-400.

2. Ronca R, Benkheil M, Mitola S, Struyf S, Liekens S Tumor angiogenesis revisited: Regulators and clinical implications. Med Res Rev 2017;1-44.

3. Cabral T, Lima LH, Polido J, Duong J, Okuda É, Oshima A, et al. Aqueous vascular endothelial growth factor and clinical outcomes correlation after single intravitreal injection of bevacizumab in patients with neovascular age - related macular degeneration. Int $\mathrm{J}$ Retin Vitr. 2017;3:4-11.

4. Izzedine H, Massard C, Spano JP, Goldwasser F, Khayat D, Soria JC. VEGF signalling inhibition-induced proteinuria: Mechanisms, significance and management. Eur J Cancer. 2010;46:439-48.

5. Lafayette RA, Mccall B, Li N, Chu L, Werner P, Das A, et al. Incidence and relevance of proteinuria in bevacizumab-treated patients: Pooled analysis from randomized controlled trials. Am J Nephrol. 2014;40:75-83.

6. Abbas A, Mirza MM, Ganti AK, Tendulkar K. Renal toxicities of targeted therapies. Target Oncol. 2015;10:487-99.

7. Lomax AJ, Hill PA, Ashley DM. Case report of interstitial nephritis induced by bevacizumab therapy for glioblastoma multiforme. J Oncol Pharm Pract. 2013;19:365-8.

8. Barakat RK, Singh N, Lal R, Verani RR, Finkel KW, Foringer JR. Interstitial nephritis secondary to bevacizumab treatment in metastatic leiomyosarcoma. Ann Pharmacother. 2007;41:707-10.

9. Hanna RM, Lopez E, Wilson J, Barathan S, Cohen AH. Minimal change disease onset observed after bevacizumab administration. Clin Kidney J. 2016;9:239-44.

10. Frangié C, Lefaucheur C, Medioni J, Jacquot C, Hill GS, Nochy D. Renal thrombotic microangiopathy caused by anti-VEGFantibody treatment for metastatic renal-cell carcinoma. Lancet Oncol. 2007;8:177-8. 
11. Uy AL, Simper NB, Champeaux AL, Perkins RM. Progressive bevacizumab-associated renal thrombotic microangiopathy. NDT Plus. 2009;2:36-39.

12. Cheungpasitporn W, Chebib FT, Cornell LD, Brodin ML, Nasr SH, Schinstock CA, et al. Intravitreal antivascular endothelial growth factor therapy may induce proteinuria and antibody mediated injury in renal allografts. Transplantation. 2015;99:2382-6.

13. Eremina V, Jefferson JA, Kowalewska J, Hochster H, Haas M, Weisstuch $J$, et al. VEGF Inhibition and Renal Thrombotic Microangiopathy. N Engl J Med. 2008;11:1129-36.

14. Gelaye B, Rondon M, Araya PR, Drug-Induced Thrombotic APM. Microangiopathy. Experience from the Oklahoma Registry and The Bloodcenter of Wisconsin. Am J Hematol. 2016;3:973-82.

15. Muto J, Kishimoto H, Katzuka Y, Kinjo M, Higashi H, Kishihara F. Thrombotic microangiopathy following chemotherapy with s-1 and cisplatin in a patient with gastric cancer: a case report. In Vivo (Brooklyn). 2017;31:439-41.

16. Sugimoto H, Hamanog Y, Charytan D, Cosgrove D, Kieran M, Sudhakar A, et al. Neutralization of circulating vascular endothelial growth factor (VEGF) by anti-VEGF antibodies and soluble VEGF receptor 1 (sFlt-1) induces proteinuria. J Biol Chem. 2003;278:12605-8.

17. Keir LS, Firth R, Aponik L, Feitelberg D, Sakimoto S, Aguilar E, et al. VEGF regulates local inhibitory complement proteins in the eye and kidney. J Clin Invest. 2017;127:199-214.

18. Zhao N, Xu Q, Wang M, Fei X, Pan Y, Chen X, et al. Mechanism of kidney injury caused by bevacizumab in rats. Int J Clin Exp Pathol. 2014;7:8675-83.

19. Gerber H-P, Wu X, Yu L, Wiesmann C, Liang XH, Lee CV, et al. Mice expressing a humanized form of VEGF-A may provide insights into the safety and efficacy of anti-VEGF antibodies. Proc Natl Acad Sci U S A. 2007;104:3478-83.

20. Meyer T, Robles-Carrillo L, Robson T, Langer F, Desai H, Davila $\mathrm{M}$, et al. Bevacizumab immune complexes activate platelets and induce thrombosis in FCGR2A transgenic mice. J Thromb Haemost. 2009;7:171-81.

21. Julien S, Biesemeier A, Schraermeyer U. In vitro induction of protein complexes between bevacizumab, VEGF-A and heparin: explanation for deposits observed on endothelial veins in monkey eyes. Br J Ophthalmol. 2013;97:511-7.

22. Trimarchi H, Barratt J, Cattran DC, Cook HT, Coppo R, Haas M, et al. Oxford Classification of IgA nephropathy 2016: an update from the IgA Nephropathy Classification Working Group. Kidney Int. 2017;91:1014-21.

23. Reagents PLA Duolink ${ }^{\circledR}$ PLA Brightfield Protocol. 2018.Available from: https://www.sigmaaldrich.com/content/dam/sigma-aldrich/ docs/promo_NOT_INDEXED/General_Information/1/duolink-plabrightfield-protocol-msig.pdf.
24. Zipfel PF, Mache C, Müller D, Licht C, Wigger M, Skerka C. DEAP-HUS: Deficiency of CFHR plasma proteins and autoantibody-positive form of hemolytic uremic syndrome. Pediatr Nephrol. 2010;25:2009-19.

25. Hackl A, Ehren R, Kirschfink M, Zipfel PF, Beck BB, Weber LT, et al. Successful discontinuation of eculizumab under immunosuppressive therapy in DEAP-HUS. Pediatr Nephrol. 2017;32:1081-7.

26. Höhne M, Frese CK, Grahammer F, Dafinger C, Ciarimboli G, Butt L, et al. Single nephron proteomes connect morphology and function in proteinuric kidney disease. Kidney Int 2018;1308-19.

27. Hughes CS, Foehr S, Garfield DA, Furlong EE, Steinmetz LM, Krijgsveld J. Ultrasensitive proteome analysis using paramagnetic bead technology. Mol Syst Biol. 2014;10:757-757.

28. Cox J, Mann M. MaxQuant enables high peptide identification rates, individualized p.p.b.-range mass accuracies and proteomewide protein quantification. Nat Biotechnol. 2008;26:1367-72.

29. www.drugbank.ca [Internet]. 2018.Available from: www.drugba nk.ca.

30. Cox J, Hein MY, Luber CA, Paron I, Nagaraj N, Mann M. Accurate Proteome-wide Label-free Quantification by Delayed Normalization and Maximal Peptide Ratio Extraction, Termed MaxLFQ. Mol Cell Proteomics. 2014;13:2513-26.

31. Tusher VG, Tibshirani R, Chu G. Significance analysis of microarrays applied to the ionizing radiation response. Proc Natl Acad Sci. 2001;98:5116-21.

32. Han MH, Kim YJ Practical application of columbia classification for focal segmental glomerulosclerosis. Biomed Res Int 2016;2016. https://doi.org/10.1155/2016/9375753.

33. NoBagchi S, Fredriksson R W-MÅ. In Situ Proximity Ligation Assay (PLA). In: Hnasko R (eds) ELISA. Methods in Molecular Biology, vol 1318. Humana Press, New York, NY, 1st ed. New York: Humana Press, 2015 https://doi.org/10.1007/978-1-49392742-5 15

34. Andeen NK, Yang H-Y, Dai D-F, MacCoss MJ, Smith KD DnaJ Homolog Subfamily B Member 9 Is a Putative Autoantigen in Fibrillary GN. J Am Soc Nephrol 2017;ASN.2017050566.

35. Buob D, Decambron M, Gnemmi V, Frimat M, Hoffmann M, Azar R, et al. Collapsing glomerulopathy is common in the setting of thrombotic microangiopathy of the native kidney. Kidney Int. 2016;90:1321-31.

36. Zipfel PF, Heinen S, Skerka C. Thrombotic microangiopathies: new insights and new challenges. Curr Opin Nephrol Hypertens. 2010;19:372-8.

37. Tanaka H, Takahashi K, Yamaguchi K, Kontani K, Motoki T, Asakura M, et al. Hypertension and Proteinuria as Predictive Factors of Effects of Bevacizumab on Advanced Breast Cancer in Japan. 2018;41:644-8. 